\title{
A RUNGE THEOREM FOR SOLUTIONS OF THE HEAT EQUATION
}

\author{
R. DIAZ
}

\begin{abstract}
Let $\Omega_{1}$ and $\Omega_{2}$ be open sets in $R^{n}$ such that $\Omega_{1} \subset \Omega_{2}$. Every solution of the heat equation on $\Omega_{1}$ admits approximation on the compact subsets of $\Omega_{1}$ by functions which satisfy the heat equation throughout $\Omega_{2}$ if and only if this topological condition is met: For every hyperplane $\pi$ in $R^{n}$ orthogonal to the time axis, every compact component of $\pi \backslash \Omega_{1}$ contains a compact component of $\pi \backslash \Omega_{2}$.
\end{abstract}

1. Introduction. If $P$ is a linear partial differential operator on $R^{n}$ and if $\Omega$ is an open set in $R^{n}$, let $P(\Omega)$ denote the kernel of the map $C^{\infty}(\Omega) \ni f \rightarrow P f \in C^{\infty}(\Omega)$. Observe that if $\Omega^{\prime}$ is an open subset of $\Omega$ then the restriction map $r: C^{\infty}(\Omega) \rightarrow$ $C^{\infty}\left(\Omega^{\prime}\right)$ carries $P(\Omega)$ to a subspace of $P\left(\Omega^{\prime}\right)$. If $r(P(\Omega))$ is dense in $P\left(\Omega^{\prime}\right)$ when $P\left(\Omega^{\prime}\right)$ carries the topology induced by $C^{\infty}\left(\Omega^{\prime}\right)$, then the pair $\left(\Omega^{\prime}, \Omega\right)$ is called a $P$-Runge pair and $\Omega^{\prime}$ is called a relative $P$-Runge domain of $\Omega$; cf. [5]. For a large class of elliptic operators Malgrange has proved that $\left(\Omega^{\prime}, \Omega\right)$ is a $P$-Runge pair if and only every compact component of $R^{n} \backslash \Omega^{\prime}$ contains a compact component of $R^{n} \backslash \Omega$ [4]. No general topological characterization of $P$-Runge pairs has been given for nonelliptic operators; however, B. F. Jones has proved recently that for the parabolic operator $H$, the heat operator on $R^{n}$, the pair $\left(\Omega, R^{n}\right)$ is an $H$-Runge pair if and only if each hyperplane in $R^{n}$ orthogonal to the time axis intersects $R^{n} \backslash \Omega$ to form a set that is free of compact components [3]. We shall generalize the theorem of Jones by characterizing all $\boldsymbol{H}$-Runge pairs.

2. Statement of results. Let $H$ denote the heat operator on $R^{n}, n \geqslant 2$, and let $\Omega_{1}$ and $\Omega_{2}$ be open sets in $R^{n}$ such that $\Omega_{1} \subset \Omega_{2}$. Our main result is

THEOREM 1. If, for each hyperplane $\pi$ in $R^{n}$ orthogonal to the time axis, every compact component of $\pi \backslash \Omega_{1}$ contains a compact component of $\pi \backslash \Omega_{2}$, then $\left(\Omega_{1}, \Omega_{2}\right)$ is an $H$-Runge pair.

Modifying the work of Jones in a straightforward fashion, we also prove the converse.

THEOREM 2. If $\left(\Omega_{1}, \Omega_{2}\right)$ is an $H$-Runge pair then, for each hyperplane $\pi$ orthogonal to the time axis, every compact component of $\pi \backslash \Omega_{1}$ contains a compact component of $\pi \backslash \Omega_{2}$.

Received by the editors June 25, 1979.

AMS (MOS) subject classifications (1970). Primary 35K05, 35A35; Secondary 35E10.

Key words and phrases. Relative $P$-Runge domains, parabolic operators, $H$-Runge pairs, nonuniqueness for the initial value problem. 
3. Proof of the main theorem. The Hahn-Banach theorem implies that $\left(\Omega_{1}, \Omega_{2}\right)$ is an $H$-Runge pair if and only if each distribution $\mu \in \mathcal{E}^{\prime}\left(\Omega_{1}\right)$ that annihilates $H\left(\Omega_{2}\right)$ also annihilates $H\left(\Omega_{1}\right)$. If $\mu$ does annihilate $H\left(\Omega_{2}\right), \mu$ annihilates in particular the exponential-polynomial members of $H\left(R^{n}\right)$, and a theorem of Malgrange implies that $\mu={ }^{\dagger} H \nu$ for some $\nu \in \mathcal{E}^{\prime}\left(R^{n}\right)$ where ${ }^{\dagger} H$ denotes the operator formally adjoint to $H$ [4]. If $\nu$ is supported in $\Omega_{1}$ there exists a cut-off function $\varphi$ such that for each $f \in H\left(\Omega_{1}\right)$ the function $\varphi f$ agrees with $f$ on a neighborhood of supp $\nu$ and $\varphi f \in$ $\mathcal{E}\left(R^{n}\right)$; consequently, supp $\nu \cap \operatorname{supp} H(\varphi f)=\varnothing$ and

$$
\langle\mu, f\rangle=\left\langle{ }^{\dagger} H \nu, f\right\rangle=\left\langle{ }^{\dagger} H \nu, \varphi f\right\rangle=\langle\nu, H(\varphi f)\rangle=0 .
$$

Thus, $\mu$ annihilates $H\left(\Omega_{1}\right)$ if $\nu$ is supported in $\Omega_{1}$. The remainder of this section deals with the complications which arise when $\nu$ is not supported in $\Omega_{1}$. We shall prove that supp $\nu \backslash \Omega_{1}$ is a compact subset of $\partial \Omega_{1}$ on which $\nu$ vanishes to infinite order; then, approximating $\nu$ by functions which are supported in $\Omega_{1}$, we shall apply (3.1) to conclude that $\mu$ does indeed annihilate $H\left(\Omega_{1}\right)$.

Let $E$ be a fundamental solution for the operator ${ }^{\dagger} H$. For each multi-index $\alpha=\left(\alpha_{1}, \alpha_{2}, \ldots, \alpha_{n}\right)$,

$$
D^{\alpha} \nu=D^{\alpha} \nu * \delta=D^{\alpha} \nu *{ }^{\dagger} H E={ }^{\dagger} H D^{\alpha} \nu * E={ }^{\dagger} H \nu * D^{\alpha} E=\mu * D^{\alpha} E .
$$

The hypoellipticity of ${ }^{\dagger} H$ implies that for each $x \in R^{n} \backslash \operatorname{supp} \mu$

$$
D^{\alpha} \nu(x)=\left\langle\mu, D^{\alpha} E(x-\cdot)\right\rangle
$$

where $D^{\alpha} E(x-\cdot)$ denotes the function $y \rightarrow D^{\alpha} E(x-y)$. Since

$$
H D^{\alpha} E(x-\cdot)={ }^{\dagger} H D^{\alpha} E(x-\cdot)=D^{\alpha}{ }^{\dagger} H E(x-\cdot)=D^{\alpha} \delta(x-\cdot),
$$

$D^{\alpha} E(x-\cdot) \in H\left(\Omega_{2}\right)$ for each $x \notin \Omega_{2}$. The hypothesis that $\mu$ annihilates $H\left(\Omega_{2}\right)$ and (3.2) imply that $\nu$ vanishes to infinite order on $R^{n} \backslash \Omega_{2}$.

Let $\pi$ be any hyperplane in $R^{n}$ orthogonal to the time axis. Since $D^{\alpha} \nu$ satisfies ${ }^{\dagger} H\left(D^{\alpha} \nu\right)=0$ on $R^{n} \backslash \operatorname{supp} \mu, D^{\alpha} \nu$ is real-analytic in the space variables on $R^{n} \backslash \operatorname{supp} \mu$. Thus, $D^{\alpha} \nu$ vanishes on every component of $\pi \backslash \operatorname{supp} \mu$ that contains an infinite order zero of $\nu$. Every unbounded component of $\pi \backslash \operatorname{supp} \mu$ contains such a zero because $\nu$ has compact support. Every bounded component of $\pi \backslash \operatorname{supp} \mu$ that contains a compact component of $\pi \backslash \Omega_{1}$ must contain a compact component of $\pi \backslash \Omega_{2}$ by the hypothesis of Theorem 1; consequently, $D^{\alpha} \nu$ vanishes on every component of $\pi \backslash$ supp $\mu$ that contains a component of $\pi \backslash \Omega_{1}$. Since $\pi$ and $\alpha$ are arbitrary, $\nu$ must vanish to infinite order throughout $R^{n} \backslash \Omega_{1}$. Clearly then supp $\nu \subset \bar{\Omega}_{1}$, and $\nu$ vanishes to infinite order on $\operatorname{supp} \nu \backslash \Omega_{1}$. The closed set $K=\operatorname{supp} \nu \backslash \Omega_{1}$ is compact because $\nu$ has compact support.

Henceforth we shall write $R^{n}$ as $R^{n-1} \times R$ so that we may distinguish between the one time variable and the $n-1$ space variables on which solutions of the heat equation depend. Let $\left\{D_{i}\right\}_{i=1}^{m}$ be a finite collection of mutually disjoint, connected open sets such that

(i) each $D_{i}$ is a product of a subset of $R^{n-1}$ and a subinterval of $R$,

(ii) $\bar{D}_{i} \cap \bar{D}_{j}$ is contained in a hyperplane orthogonal to the time axis and has finitely many components for $i \neq j$; and 
(iii) $F=\cup_{i=1}^{m} \bar{D}_{i}$ is a compact neighborhood of $K$ disjoint from supp $\mu$.

Let $\varphi$ be a cut-off function supported in the interior of $F$ such that $\varphi \equiv 0$ on a neighborhood of each component of $\bar{D}_{i} \cap \bar{D}_{j}$ disjoint from $K$ and such that $\varphi \equiv 1$ on a neighborhood of $K$. Finally, let $Z=\{x \notin \operatorname{supp} \mu \mid \nu$ vanishes to infinite order at $x$ \}. Any $\bar{D}_{i} \cap \bar{D}_{j}$ has its components contained in a copy of $R^{n-1}$ when $i \neq j$; and since $\nu$ is real-analytic in the space variables on a neighborhood of $\bar{D}_{i} \cap \bar{D}_{j}, \nu$ vanishes to infinite order throughout any component of $\bar{D}_{i} \cap \bar{D}_{j}$ meeting $K$. Thus $\varphi \nu$ vanishes to infinite order throughout $\bar{D}_{i} \cap \bar{D}_{j}$ when $i \neq j$. Since each boundary point of $D_{i}$ is either contained in some $\bar{D}_{j}$ or is a boundary point of $F, \varphi \nu$ vanishes to infinite order throughout the boundary of $D_{i}$. Clearly then $\varphi \nu$ vanishes to infinite order throughout the boundary of each component of $D_{i} \backslash Z$.

Now, for each $f \in H\left(\Omega_{1}\right)$,

$$
\begin{aligned}
\langle\mu, f\rangle & =\langle(1-\varphi) \mu+\varphi \mu, f\rangle, \\
& =\left\langle{ }^{\dagger} H[(1-\varphi) \nu], f\right\rangle+\left\langle{ }^{\dagger} H(\varphi \nu), f\right\rangle, \\
& =\left\langle{ }^{\dagger} H(\varphi \nu), f\right\rangle,
\end{aligned}
$$

since $H f \equiv 0$ on a neighborhood of $\operatorname{supp}(1-\varphi) \nu$. Recall that $\varphi \nu$ is smooth and supported in $F$. Evidently, then,

$$
\langle\mu, f\rangle=\int_{F} f^{\dagger} H(\varphi v)=\sum_{i=1}^{m} \int_{D_{i}} f^{\dagger} H(\varphi \nu)=\sum_{i=1}^{m} \int_{D_{i} \backslash Z} f^{\dagger} H(\varphi \nu) .
$$

Let $A \times(a, b)$ be an arbitrary component of some $D_{i} \backslash Z$, where $A \subset R^{n-1}$ and $(a, b) \subset R$. We shall prove that $\int_{A \times(a, b)} f^{\dagger} H(\varphi \nu)=0$, from which it follows that $\mu$ annihilates $H\left(\Omega_{1}\right)$.

Note that since $\varphi \nu$ vanishes to infinite order throughout the boundary of $A \times(a, b)$ the function $\overline{\varphi \nu}$ which agrees with $\varphi \nu$ on $A \times(a, b)$ and which is zero outside $A \times(a, b)$ is smooth throughout $R^{n-1} \times R$. Composing $\overline{\varphi \nu}$ with a translation of $R^{n-1} \times R$, we may assume that $(0,0) \in A \times(a, b)$. Now let $B \times(c, d)$ be a connected open neighborhood of $\bar{A} \times[a, b]$ such that $\bar{B} \times[c, d]$ is a subset of $\left(R^{n-1} \times R\right) \backslash \operatorname{supp} \mu$. Clearly $B \times(a, b)$ is disjoint from $Z$ because $A \times(a, b)$ is disjoint from $Z$. For each $t>1$ the dilation $T_{t}: R^{n-1} \times R \rightarrow R^{n-1} \times R$ defined by $(\xi, \tau) \rightarrow T_{t}(\xi, \tau)=\left(t \xi, t^{2} \tau\right)$ is such that $R^{n-1} \times[a, b] \subset T_{t}\left(R^{n-1} \times(a, b)\right)$; thus, $T_{t}^{*}(\overline{\varphi \nu})=\overline{\varphi \nu} \circ T_{t}$ is supported in $R^{n-1} \times(a, b)$. For all $t$ sufficiently close to 1 , $T_{t}(\operatorname{supp} \overline{\varphi \nu})$ is contained in $B \times(c, d)$. For such $t$ we have supp $T_{t}^{*}(\overline{\varphi \nu})=$ $T_{t}^{-1}(\operatorname{supp} \overline{\varphi \nu}), \subset B \times(c, d) \cap R^{n-1} \times(a, b), \subset B \times(a, b)$. Thus supp $T_{t}^{*}(\overline{\varphi \nu})$ is disjoint from $Z$. In particular, supp $T_{t}^{*}(\overline{\varphi v})$ is disjoint from $R^{n} \backslash \Omega_{1}$. Since ${ }^{\dagger} H T_{t}^{*}(\overline{\varphi v})$ $=t^{2} T_{t}^{*}\left({ }^{\dagger} H \overline{\varphi v}\right)$

$$
\lim _{t \rightarrow 1^{+}}\left\langle f,{ }^{\dagger} H T_{t}^{*}(\overline{\varphi \nu})\right\rangle=\left\langle f,{ }^{\dagger} H \overline{\varphi \nu}\right\rangle=\int_{A \times(a, b)} f^{\dagger} H(\varphi \nu) .
$$

On the other hand, since ${ }^{\dagger} H T_{t}^{*}(\overline{\varphi \nu})$ is supported in $\Omega_{1}$,

$$
\left\langle f,{ }^{\dagger} H T_{t}^{*}(\overline{\varphi \nu})\right\rangle=\left\langle H f, T_{t}^{*}(\overline{\varphi \nu})\right\rangle=\left\langle 0, T_{t}^{*}(\overline{\varphi v})\right\rangle=0 .
$$

Thus, $\int_{A \times(a, b)} f^{\dagger} H(\overline{\varphi \nu})=0$ and $\mu$ annihilates $H\left(\Omega_{1}\right)$. Q.E.D. 
4. The converse to the main theorem. Our proof that the topological condition on $\Omega_{1}$ and $\Omega_{2}$ is necessary for $\left(\Omega_{1}, \Omega_{2}\right)$ to be an $H$-Runge pair is a modification of the proof given in [3] for the special case $\Omega_{2}=R^{n}$. This proof requires the Tychonoff counterexample to uniqueness to the initial-value problem for the heat equation, which is a nonzero element of $H\left(R^{n}\right)$ supported in a strip bounded by two hyperplanes orthogonal to the time axis. The existence of such a function is proved in [2].

We shall prove that if for some hyperplane $\pi$ orthogonal to the time axis some compact component of $\pi \backslash \Omega_{1}$ is contained in $\Omega_{2}$, then there exists a $\mu \in \mathcal{E}^{\prime}\left(\Omega_{1}\right)$ such that $\mu$ annihilates $H\left(\Omega_{2}\right)$ but such that $\mu$ does not annihilate $H\left(\Omega_{1}\right)$; the Hahn-Banach theorem then implies that $H\left(\Omega_{2}\right)$ is not dense in $H\left(\Omega_{1}\right)$. We may assume that $\pi=R^{n-1} \times\{0\}$ and identify $\pi$ with $R^{n-1}$. Now, there exists an open set $G$ in $R^{n-1}$ contained in $\Omega_{2}$ and a nonempty compact subset $K$ of $\pi \backslash \Omega_{1}$ such that $G \backslash \Omega_{1}=K[1]$. Choose $\varphi \in \mathscr{D}(G)$ such that $\varphi \equiv 1$ on a neighborhood of $K$ in $R^{n-1}$. There exists a $\delta>0$ such that $\operatorname{supp} \varphi \times[-\delta, \delta] \subset \Omega_{2}$ and such that $\operatorname{supp}\|\nabla \varphi\| \times[-\delta, \delta] \subset \Omega_{1}$, where $\nabla$ is the $(n-1)$-dimensional gradient. By suitably rescaling the Tychonoff counterexample to uniqueness and reversing the sign of the time variable, we can construct a function $g$ supported in $R^{n-1} \times[-\delta, \delta]$ such that ${ }^{\dagger} H g=0$ throughout $R^{n}$ and $g$ is nonzero at some point $x_{0}$ in $K$. The function ${ }^{\dagger} H(\varphi g)$ is supported in supp $\|\nabla \varphi\| \times[-\delta, \delta] \subset \Omega_{1}$, and $\varphi g$ is supported in supp $\varphi \times$ $[-\delta, \delta] \subset \Omega_{2}$. For each $f \in H\left(\Omega_{2}\right)$ one has

$$
\left\langle{ }^{\dagger} H(\varphi g), f\right\rangle=\langle g, \varphi H f\rangle=\langle g, 0\rangle=0 .
$$

However, if $E$ is any fundamental solution for the heat equation then $E\left(\cdot-x_{0}\right) \in$ $H\left(\Omega_{1}\right)$, yet

$$
\left\langle{ }^{\dagger} H(\varphi g), E\left(\cdot-x_{0}\right)\right\rangle=\left\langle\varphi g, H E\left(\cdot-x_{0}\right)\right\rangle=\left\langle\varphi g, \delta\left(\cdot-x_{0}\right)\right\rangle=g\left(x_{0}\right) \neq 0 .
$$

Thus $\mu={ }^{\dagger} H(\varphi g)$ annihilates $H\left(\Omega_{2}\right)$ but does not annihilate $H\left(\Omega_{1}\right)$; and $H\left(\Omega_{2}\right)$ is not dense in $H\left(\Omega_{1}\right)$. Q.E.D.

I wish to thank Professor B. Frank Jones, whose lectures on partial differential equations and work on the Runge problem made this paper possible.

\section{REFERENCES}

1. R. Harvey, J. Polking and R. O. Wells, Jr., Complex analysis, (in preparation).

2. G. Hellwig, Partial differential equations: an introduction, Blaisdell, Waltham, Mass., 1964. MR 30 \#3286.

3. B. F. Jones, An approximation theorem of Runge type for solutions of the heat equation, Proc. Amer. Math. Soc. 52 (1975), 289-292. MR 52 \#8654.

4. B. Malgrange, Existence et approximation des solutions des équations aux dérivées partielles et des équations de convolution, Ann. Inst. Fourier (Grenoble) 6 (1955/56), 271-355. MR 19, 280.

5. F. Treves, Locally convex spaces and linear partial differential equations, Die Grundlehren der Mathematischen Wissenschaften, Band 146, Springer-Verlag, New York, 1967.

Department of Mathematics, Rice Universtry, Houston, Texas 77001

Current address: Department of Mathematics, Princeton University, Princeton, New Jersey 08540 